\title{
On the Second-order Slip and Jump Coefficients for the General Theory of Slip Flow
}

$\operatorname{AUTHOR}(\mathrm{S})$ :

Takata, Shigeru; Hattori, Masanari

\section{CITATION:}

Takata, Shigeru ...[et al]. On the Second-order Slip and Jump Coefficients for the General Theory of Slip Flow. AIP Conference Proceedings: 28th International Symposium on Rarefied Gas Dynamics 2012 2012, 1501: 59-66

ISSUE DATE:

2012-11-27

URL:

http://hdl.handle.net/2433/187981

\section{RIGHT:}

(C) 2012 American Institute of Physics. This article may be downloaded for personal use only. Any other use requires prior permission of the author and the American Institute of Physics. 


\title{
On the Second-order Slip and Jump Coefficients for the General Theory of Slip Flow
}

\author{
Shigeru Takata*,† and Masanari Hattori* \\ * Department of Mechanical Engineering and Science, Kyoto University, Kyoto 606-8501, Japan \\ ${ }^{\dagger}$ Advanced Research Institute of Fluid Science and Engineering, Kyoto University, Kyoto 606-8501, Japan
}

\begin{abstract}
The general theory of slip flow established in the late 1960s is revisited. For a long time, the complete set of data of the slip and jump coefficients up to the second order of the small Knudsen number have been available only for the Bhatnagar-Gross-Krook model. The present paper provides the complete set of data of those coefficients for a hard-sphere gas on the diffuse reflection boundary. The data are obtained by using the general identities that have been deduced from recently developed symmetry arguments. A few simple application examples are also presented.

Keywords: slip flow, second-order slip, slightly rarefied gas, kinetic theory, Boltzmann equation, Poiseuille flow, thermal transpiration, drag, thermophoresis

PACS: $47.45 . \mathrm{Gx}, 47.45 .-\mathrm{n}, 47.61 . \mathrm{Cb}, 51.10 .+\mathrm{y}$
\end{abstract}

\section{INTRODUCTION}

Recent development of micro device technologies enhances the research activity of gas flows in the slip-flow regime, both numerically and experimentally. The general theory of slip flow itself has been established with a firm foundation in 1960s and 1970s on the basis of the Bhatnagar-Gross-Krook (BGK or Boltzmann-Krook-Welander [1, 2]) model [3] and later on the basis of the original Boltzmann equation [4]. The slip and jump conditions up to the second order of the small Knudsen number, which are recent concerns of many researchers (e.g., $[5,6,7,8])$, have also been clarified at such an early stage. They are compiled and can be found in $[9,10]$.

For a long time, the specific values of the second-order slip and jump coefficients occurring in that theory have been available only partially, except for the BGK model with the diffuse reflection condition. In the present work, we will take a step forward to provide all the values of those coefficients for a hard-sphere gas with the diffuse reflection condition. The symmetry argument recently developed by the first author [11] makes this step rather easy, compared with a straightforward numerical approach.

\section{GENERAL THEORY OF SLIP FLOW}

Let us denote by $L x_{i}$ the space coordinates, by $p_{0}(1+P)$ and $T_{0}(1+\tau)$ the pressure and temperature of the gas, by $\left(2 R T_{0}\right)^{1 / 2} u_{i}$ the flow velocity, and by $\left(2 R T_{0}\right)^{1 / 2} u_{w i}$ and $T_{0}\left(1+\tau_{w}\right)$ the velocity and temperature of the boundary. Here $L$ is the characteristic length of the system, $R$ is the specific gas constant, $p_{0}$ and $T_{0}$ are the pressure and temperature in the reference equilibrium state at rest. We also denote by $n_{i}$ the unit normal to the boundary, pointed to the gas, and by $t_{i}$ an arbitrary unit vector tangential to the boundary. We assume that the boundary does not deform and thus $u_{w i} n_{i}=0$. The slip-flow theory describes the asymptotic behavior of the gas for small Knudsen numbers. Here the Knudsen number $\mathrm{Kn}$ is defined by $\mathrm{Kn}=\ell_{0} / L$ with $\ell_{0}$ being the mean-free path of a molecule at the reference equilibrium state. ${ }^{1}$ In the present paper, we use the notation $\varepsilon$ defined by $\varepsilon=(\sqrt{\pi} / 2) \mathrm{Kn}$, instead of $\mathrm{Kn}$, to emphasize its smallness.

According to the general theory of slip flow $[9,10]$, the behavior of a slightly rarefied gas with a small Reynolds number can be described in the bulk of the domain by the Stokes set of equations with slip and jump boundary conditions; in the layer with the thickness of a few mean free paths, which is adjacent to the boundary and is called the Knudsen layer, this fluid-dynamical description (to be referred to fluid-dynamical part) is necessary to be corrected

\footnotetext{
${ }^{1}$ For the BGK model, $\ell_{0}=(2 / \sqrt{\pi})\left(2 R T_{0}\right)^{1 / 2} /\left(A_{c} \rho_{0}\right)$, where $\rho_{0}=p_{0} / R T_{0}$ (the reference density) and $A_{c} \rho_{0}$ is the collision frequency $\left(A_{c}\right.$ is a positive constant). For a hard-sphere gas, $\ell_{0}=\left(\sqrt{2} \pi d^{2}\left(\rho_{0} / m\right)\right)^{-1}$, where $d$ and $m$ are the diameter and mass of a molecule.
}

28th International Symposium on Rarefied Gas Dynamics 2012

AIP Conf. Proc. 1501, 59-66 (2012); doi: 10.1063/1.4769473

(C) 2012 American Institute of Physics 978-0-7354-1115-9/\$30.00 
(the Knudsen-layer correction). The slip and jump conditions and the Knudsen-layer correction are required at the level of the first and higher orders of the Knudsen number. This is a classical result derived by a systematic asymptotic analysis of the linearized Boltzmann equation for small Knudsen number. The primary concern of the present paper is the fluid-dynamical part. The Knudsen-layer corrections will be rarely discussed or mentioned hereafter.

We shall tell apart the fluid-dynamical part and the Knudsen-layer correction by putting subscript $\mathrm{G}$ and $\mathrm{K}$ to the notation of quantities respectively. Each quantity, say $h=h_{\mathrm{G}}+h_{\mathrm{K}}\left(h=P, \tau, u_{i}\right.$, etc.), is expanded in a power series of $\varepsilon$ as $h_{\mathrm{G}}=h_{\mathrm{G}}^{(0)}+h_{\mathrm{G}}^{(1)} \varepsilon+h_{\mathrm{G}}^{(2)} \varepsilon^{2} \cdots$ and $h_{\mathrm{K}}=h_{\mathrm{K}}^{(1)} \varepsilon+h_{\mathrm{K}}^{(2)} \varepsilon^{2} \cdots$. Note that the expansion of $h_{\mathrm{K}}$ starts from $O(\varepsilon)$ (see the previous paragraph). Then, the above mentioned Stokes set of equations and the slip and jump boundary conditions over a smooth solid body are written as follows:

Stokes set of equations

$$
\frac{\partial P_{\mathrm{G}}^{(0)}}{\partial x_{i}}=0, \quad \frac{\partial u_{\mathrm{G} i}^{(m)}}{\partial x_{i}}=0, \quad \gamma_{1} \frac{\partial^{2} u_{\mathrm{G} i}^{(m)}}{\partial x_{j}^{2}}=\frac{\partial P_{\mathrm{G}}^{(m+1)}}{\partial x_{i}}, \quad \frac{\partial^{2} \tau_{\mathrm{G}}^{(m)}}{\partial x_{j}^{2}}=0, \quad(m=0,1,2, \ldots),
$$

Slip and jump boundary conditions [up to $\left.O\left(\varepsilon^{2}\right)\right]$

the leading order (non-slip and non-jump)

$$
u_{\mathrm{G} i}^{(0)}=u_{w i}^{(0)}, \quad \tau_{\mathrm{G}}^{(0)}=\tau_{w}^{(0)},
$$

the first order

$$
\begin{aligned}
& u_{\mathrm{G} i}^{(1)} n_{i}=0, \quad u_{\mathrm{G} i}^{(1)} t_{i}=u_{w i}^{(1)} t_{i}-k_{0}\left(\frac{\partial u_{\mathrm{G} i}^{(0)}}{\partial x_{j}}+\frac{\partial u_{\mathrm{G} j}^{(0)}}{\partial x_{i}}\right) n_{i} t_{j}-K_{1} \frac{\partial \tau_{\mathrm{G}}^{(0)}}{\partial x_{i}} t_{i}, \\
& \tau_{\mathrm{G}}^{(1)}=\tau_{w}^{(1)}+d_{1} \frac{\partial \tau_{\mathrm{G}}^{(0)}}{\partial x_{i}} n_{i},
\end{aligned}
$$

the second order

$$
\begin{aligned}
u_{\mathrm{G} i}^{(2)} t_{i}= & u_{w i}^{(2)} t_{i}-k_{0}\left(\frac{\partial u_{\mathrm{G} i}^{(1)}}{\partial x_{j}}+\frac{\partial u_{\mathrm{G} j}^{(1)}}{\partial x_{i}}\right) n_{i} t_{j}-K_{1} \frac{\partial \tau_{\mathrm{G}}^{(1)}}{\partial x_{i}} t_{i}-a_{1}\left[\frac{\partial}{\partial x_{k}}\left(\frac{\partial u_{\mathrm{G} i}^{(0)}}{\partial x_{j}}+\frac{\partial u_{\mathrm{G} j}^{(0)}}{\partial x_{i}}\right)\right] n_{j} n_{k} t_{i} \\
& -a_{2} \bar{\kappa}\left(\frac{\partial u_{\mathrm{G} i}^{(0)}}{\partial x_{j}}+\frac{\partial u_{\mathrm{G} j}^{(0)}}{\partial x_{i}}\right) n_{i} t_{j}-a_{3} \kappa_{i j}\left(\frac{\partial u_{\mathrm{G} k}^{(0)}}{\partial x_{j}}+\frac{\partial u_{\mathrm{G} j}^{(0)}}{\partial x_{k}}\right) n_{k} t_{i} \\
& -\left(a_{4}-d_{1} K_{1}\right) \frac{\partial^{2} \tau_{\mathrm{G}}^{(0)}}{\partial x_{i} \partial x_{j}} n_{i} t_{j}-a_{5} \bar{\kappa} \frac{\partial \tau_{\mathrm{G}}^{(0)}}{\partial x_{i}} t_{i}-\left(a_{6}-d_{1} K_{1}\right) \kappa_{i j} \frac{\partial \tau_{\mathrm{G}}^{(0)}}{\partial x_{j}} t_{i}, \\
u_{\mathrm{G} i}^{(2)} n_{i}= & -2 b_{1} \frac{\partial^{2} u_{\mathrm{G} i}^{(0)}}{\partial x_{k} \partial x_{j}} n_{i} n_{j} n_{k}-b_{2}\left(\frac{\partial^{2} \tau_{\mathrm{G}}^{(0)}}{\partial x_{i} \partial x_{j}} n_{i} n_{j}+2 \bar{\kappa} \frac{\partial \tau_{\mathrm{G}}^{(0)}}{\partial x_{i}} n_{i}\right), \\
\tau_{\mathrm{G}}^{(2)}= & \tau_{w}^{(2)}+d_{1} \frac{\partial \tau_{\mathrm{G}}^{(1)}}{\partial x_{i}} n_{i}+2 d_{4} \frac{\partial^{2} u_{\mathrm{G} i}^{(0)}}{\partial x_{k} \partial x_{j}} n_{i} n_{j} n_{k}+d_{3} \frac{\partial^{2} \tau_{\mathrm{G}}^{(0)}}{\partial x_{i} \partial x_{j}} n_{i} n_{j}+d_{5} \bar{\kappa} \frac{\partial \tau_{\mathrm{G}}^{(0)}}{\partial x_{i}} n_{i},
\end{aligned}
$$

where $\gamma_{1}$ is the dimensionless viscosity ${ }^{2}$, which is a constant depending on the molecular model (see Table 2 in the next section), while $k_{0}, K_{1}, a_{1} \sim a_{6}, b_{1}, b_{2}, d_{1}$, and $d_{3} \sim d_{5}$ are the slip and jump coefficients, which are a constant depending on both the molecular model and the kinetic boundary condition (i.e., the model of gas-surface interaction). $\kappa_{i j} / L$ and $\bar{\kappa} / L$ are respectively the curvature matrix and mean curvature of the boundary. ${ }^{3}$ The system (1)-(7) determines the

\footnotetext{
2 The viscosity $\mu$ at the reference state is given by $\mu=\gamma_{1} p_{0}\left(2 R T_{0}\right)^{-1 / 2} L \varepsilon$.

${ }^{3} \kappa_{i j} / L$ and $\bar{\kappa} / L$ are defined by $\kappa_{i j}=\kappa_{1} m_{i} m_{j}+\kappa_{2} \ell_{i} \ell_{j}$ and $\bar{\kappa}=\left(\kappa_{1}+\kappa_{2}\right) / 2$, where $\kappa_{1} / L$ and $\kappa_{2} / L$ are the principal curvatures of the boundary and $m_{i}$ and $\ell_{i}$ are the direction cosines of the principal directions corresponding to $\kappa_{1} / L$ and $\kappa_{2} / L$. Here $\kappa_{1}$ and $\kappa_{2}$ are taken negative when the corresponding center of curvature lies on the gas side.
} 
TABLE 1. Slip and jump coefficients for the BGK model and the diffuse reflection condition (the data taken from $[10,9])$.

\begin{tabular}{|c|c|c|c|c|c|c|c|c|c|}
\hline \multicolumn{2}{|c|}{ first-order slip \& jump } & \multicolumn{4}{|c|}{ second-order slip } & \multicolumn{4}{|c|}{ second-order jump } \\
\hline$k_{0}$ & -1.01619 & $a_{1}$ & 0.76632 & $a_{4}$ & 0.27922 & $d_{3}$ & 0 & $b_{1}$ & 0.11684 \\
\hline$K_{1}$ & -0.38316 & $a_{2}$ & 0.50000 & $a_{5}$ & 0.26693 & $d_{4}$ & 0.11169 & $b_{2}$ & 0.26693 \\
\hline$d_{1}$ & 1.30272 & $a_{3}$ & -0.26632 & $a_{6}$ & -0.76644 & $d_{5}$ & 1.82181 & & \\
\hline
\end{tabular}

behavior of the bulk gas from the lowest order. Namely, the first equation of (1) means the uniform pressure at the leading order. Equation (1) for $m=0$ with (2) determines the leading order of temperature and flow velocity, together with the first order of pressure; Equation (1) for $m=1$ with (3) and (4) determine the first order of temperature and flow velocity, together with the second order of pressure; and so on.

If we sum up the terms in the above system, the behavior of the bulk gas is seen to be described correctly up to $O\left(\varepsilon^{2}\right)$ as follows:

$$
\frac{\partial u_{\mathrm{G} i}}{\partial x_{i}}=0, \quad \varepsilon \gamma_{1} \frac{\partial^{2} u_{\mathrm{G} i}}{\partial x_{j}^{2}}=\frac{\partial P_{\mathrm{G}}}{\partial x_{i}}, \quad \frac{\partial^{2} \tau_{\mathrm{G}}}{\partial x_{j}^{2}}=0,
$$

with the slip and jump conditions

$$
\begin{aligned}
u_{\mathrm{G} i} t_{i}= & u_{w i} t_{i}-\varepsilon k_{0}\left(\frac{\partial u_{\mathrm{G} i}}{\partial x_{j}}+\frac{\partial u_{\mathrm{G} j}}{\partial x_{i}}\right) n_{i} t_{j}-\varepsilon K_{1} \frac{\partial \tau_{\mathrm{G}}}{\partial x_{i}} t_{i}-\varepsilon^{2} a_{1}\left[\frac{\partial}{\partial x_{k}}\left(\frac{\partial u_{\mathrm{G} i}}{\partial x_{j}}+\frac{\partial u_{\mathrm{G} j}}{\partial x_{i}}\right)\right] n_{j} n_{k} t_{i} \\
& -\varepsilon^{2} a_{2} \bar{\kappa}\left(\frac{\partial u_{\mathrm{G} i}}{\partial x_{j}}+\frac{\partial u_{\mathrm{G} j}}{\partial x_{i}}\right) n_{i} t_{j}-\varepsilon^{2} a_{3} \kappa_{i j}\left(\frac{\partial u_{\mathrm{G} k}}{\partial x_{j}}+\frac{\partial u_{\mathrm{G} j}}{\partial x_{k}}\right) n_{k} t_{i} \\
& -\varepsilon^{2}\left(a_{4}-d_{1} K_{1}\right) \frac{\partial^{2} \tau_{\mathrm{G}}}{\partial x_{i} \partial x_{j}} n_{i} t_{j}-\varepsilon^{2} a_{5} \bar{\kappa} \frac{\partial \tau_{\mathrm{G}}}{\partial x_{i}} t_{i}-\varepsilon^{2}\left(a_{6}-d_{1} K_{1}\right) \kappa_{i j} \frac{\partial \tau_{\mathrm{G}}}{\partial x_{j}} t_{i}, \\
u_{\mathrm{G} i} n_{i}= & -2 \varepsilon^{2} b_{1} \frac{\partial^{2} u_{\mathrm{G} i}}{\partial x_{j} \partial x_{k}} n_{i} n_{j} n_{k}-\varepsilon^{2} b_{2}\left(\frac{\partial^{2} \tau_{\mathrm{G}}}{\partial x_{i} \partial x_{j}} n_{i} n_{j}+2 \bar{\kappa} \frac{\partial \tau_{\mathrm{G}}}{\partial x_{i}} n_{i}\right), \\
\tau_{\mathrm{G}}=\tau_{w} & +\varepsilon d_{1} \frac{\partial \tau_{\mathrm{G}}}{\partial x_{i}} n_{i}+2 \varepsilon^{2} d_{4} \frac{\partial^{2} u_{\mathrm{G} i}}{\partial x_{j} \partial x_{k}} n_{i} n_{j} n_{k}+\varepsilon^{2} d_{3} \frac{\partial^{2} \tau_{\mathrm{G}}}{\partial x_{i} \partial x_{j}} n_{i} n_{j}+\varepsilon^{2} d_{5} \bar{\kappa} \frac{\partial \tau_{\mathrm{G}}}{\partial x_{i}} n_{i} .
\end{aligned}
$$

The system (8)-(11) might be preferred as a concise form of (1)-(7), especially for numerical computations.

In order to apply the theory to specific problems, a complete set of data of $k_{0}, K_{1}, a_{1} \sim a_{6}, b_{1}, b_{2}, d_{1}$, and $d_{3} \sim d_{5}$ is desired. However, it is available only for the BGK model with the diffuse reflection condition ([3, 9]; see Table 1). Even for a hard-sphere gas with the diffuse reflection condition, only a partial set of data (i.e., $k_{0}, K_{1}, a_{4}, b_{1}, b_{2}, d_{1}$, and $d_{4}$ ) has been available $[12,13,14,15]$, thus having restricted the application of the theory to the level of $O(\varepsilon)$.

In the next section, we provide a complete set of numerical data of the slip and jump coefficients for a hard-sphere gas on the diffuse reflection boundary, which was obtained by using the symmetric relation developed in [11].

\section{IDENTITIES AMONG THE SLIP AND JUMP COEFFICIENTS}

Each slip and jump coefficient is related to a specific thermal or fluid-dynamical state (shear stress, temperature gradient, thermal stress, etc.) of the bulk gas at the boundary and is determined (together with the solution, namely the velocity distribution function) through the analysis of the corresponding half-space problem of the linearized Boltzmann equation with/without a source term. This half-space problem is generically written as

$$
\begin{aligned}
& \zeta_{n} \frac{\partial \phi^{\alpha}}{\partial \eta}=\mathscr{L}\left[\phi^{\alpha}\right]+I^{\alpha}(\eta, \zeta), \\
& \phi^{\alpha}=\widetilde{\mathscr{K}}\left[-2 \zeta_{j}\left(\delta_{i j}-n_{i} n_{j}\right) b_{i}^{\alpha}-|\zeta|^{2} c^{\alpha}+g^{\alpha}(\boldsymbol{\zeta})\right]+\mathscr{K}\left[\phi^{\alpha}\right], \quad \zeta_{n}>0, \eta=0, \\
& \phi^{\alpha} \rightarrow 0 \text { as } \eta \rightarrow \infty,
\end{aligned}
$$

where $\zeta_{n}=\zeta_{i} n_{i}, \eta$ is the (stretched dimensionless) coordinate in the direction of $n_{i}, \mathscr{L}$ is the linearized collision operator, $\mathscr{K}$ is the locally isotropic reflection operator on the boundary, $\widetilde{\mathscr{K}}[f]=f-\mathscr{K}[f], I^{\alpha}$ and $g^{\alpha}$ are a given 
function, $\delta_{i j}$ is the Kronecker delta, superscript $\alpha$ is the label for telling apart the elemental problems, and $b_{i}^{\alpha}$ and $c^{\alpha}$ correspond to the slip coefficient of tangential velocity and the jump coefficient of temperature, respectively. For instance, $\mathscr{L}$ is given for the BGK model by

$$
\mathscr{L}\left[\phi^{\alpha}\right]=\left\langle\phi^{\alpha}\right\rangle+2 \zeta_{i}\left\langle\zeta_{i} \phi^{\alpha}\right\rangle+\frac{2}{3}\left(|\boldsymbol{\zeta}|^{2}-\frac{3}{2}\right)\left\langle\left(|\boldsymbol{\zeta}|^{2}-\frac{3}{2}\right) \phi^{\alpha}\right\rangle,
$$

and for a hard-sphere gas by

$$
\begin{aligned}
& \mathscr{L}\left[\phi^{\alpha}\right]=-v(|\boldsymbol{\zeta}|) \phi^{\alpha}(\boldsymbol{\zeta})+\int k(\boldsymbol{\zeta}, \boldsymbol{\xi}) \phi^{\alpha}(\boldsymbol{\xi}) d \boldsymbol{\xi}, \\
& v(z)=\frac{1}{2 \sqrt{2}}\left[\exp \left(-z^{2}\right)+\left(2 z+\frac{1}{z}\right) \int_{0}^{z} \exp \left(-y^{2}\right) d y\right], \\
& k(\boldsymbol{\zeta}, \boldsymbol{\xi})=\frac{1}{\sqrt{2} \pi|\boldsymbol{\zeta}-\boldsymbol{\xi}|} \exp \left(-|\boldsymbol{\xi}|^{2}+\frac{|\boldsymbol{\xi} \times \boldsymbol{\zeta}|^{2}}{|\boldsymbol{\xi}-\boldsymbol{\zeta}|^{2}}\right)-\frac{|\boldsymbol{\zeta}-\boldsymbol{\xi}|}{2 \sqrt{2} \pi} \exp \left(-|\boldsymbol{\xi}|^{2}\right),
\end{aligned}
$$

where

$$
\langle\Phi\rangle(\eta)=\pi^{-3 / 2} \int \Phi(\eta, \zeta) \exp \left(-|\zeta|^{2}\right) d \zeta .
$$

For the diffuse reflection condition, $\mathscr{K}$ is given by

$$
\mathscr{K}\left[\phi^{\alpha}\right]=2 \pi^{-1} \int_{\zeta_{n}^{*}<0}\left|\zeta_{n}^{*}\right| \phi^{\alpha}\left(\zeta^{*}\right) \exp \left(-\left|\zeta^{*}\right|^{2}\right) d \zeta^{*}, \quad \zeta_{n}>0 .
$$

By applying the symmetric relation developed in [11], we obtain the following identity between the half-space problem for $\phi^{\alpha}[(12)$ with $\alpha=\alpha]$ and that for $\phi^{\beta}[(12)$ with $\alpha=\beta]$ :

$$
\begin{aligned}
\left.\left\langle\zeta_{n}\left(2 \zeta_{j}\left(\delta_{i j}-n_{i} n_{j}\right) b_{i}^{\beta}-|\zeta|^{2} c^{\beta}\right)\left(\phi^{\alpha}-g^{\alpha}\right)\right\rangle\right|_{\eta=0}+\left.\left\langle\zeta_{n} g^{\beta-}\left(\phi^{\alpha}-\frac{1}{2} g^{\alpha}\right)\right\rangle\right|_{\eta=0}-\int_{0}^{\infty}\left\langle I^{\beta-} \phi^{\alpha}\right\rangle d \eta \\
=\left.\left\langle\zeta_{n}\left(2 \zeta_{j}\left(\delta_{i j}-n_{i} n_{j}\right) b_{i}^{\alpha}-|\zeta|^{2} c^{\alpha}\right)\left(\phi^{\beta}-g^{\beta}\right)\right\rangle\right|_{\eta=0}+\left.\left\langle\zeta_{n} g^{\alpha-}\left(\phi^{\beta}-\frac{1}{2} g^{\beta}\right)\right\rangle\right|_{\eta=0}-\int_{0}^{\infty}\left\langle I^{\alpha-} \phi^{\beta}\right\rangle d \eta
\end{aligned}
$$

where superscript "_" means that $\Phi^{-}(\cdot, \zeta)=\Phi(\cdot,-\zeta)$. The specific form of $I^{\alpha}$ and $g^{\alpha}$ for various elemental halfspace problems can be found in [16]. Here we simply summarize the identities between different slip and jump coefficients that have been obtained from (13) for various pairs of those half-space problems:

$$
\begin{aligned}
K_{1}= & \frac{1}{2 \gamma_{1}}\left(-\gamma_{3}+\int_{0}^{\infty}\left\langle\left(|\zeta|^{2}-\zeta_{n}^{2}\right)\left(|\zeta|^{2}-\frac{5}{2}\right) \phi^{\mathrm{S}}\right\rangle d \eta\right), \\
a_{4}= & d_{1} K_{1}-\frac{1}{\gamma_{1}} k_{0}\left(\gamma_{3}+\int_{0}^{\infty}\left\langle|\zeta|^{2} \phi^{\mathrm{T}}\right\rangle d \eta\right)+\frac{1}{\gamma_{1}} \int_{0}^{\infty} \eta\left(\left\langle|\zeta|^{2} \phi^{\mathrm{T}}\right\rangle+\left\langle\left(|\zeta|^{2}-\zeta_{n}^{2}\right)\left(|\zeta|^{2}-\frac{5}{2}\right) \phi^{\mathrm{S}}\right\rangle\right) d \eta \\
& +\frac{1}{2 \gamma_{1}} \int_{0}^{\infty}\left\langle\left(|\zeta|^{2}-\zeta_{n}^{2}\right)\left(\zeta_{n} B+\phi^{\mathrm{S}-}\right) \phi^{\mathrm{T}}\right\rangle d \eta, \\
a_{1}= & \frac{\gamma_{6}}{\gamma_{1}}+\frac{1}{2} \int_{0}^{\infty}\left\langle\left(|\zeta|^{2}-\zeta_{n}^{2}\right) \phi^{\mathrm{S}}\right\rangle d \eta, \\
a_{3}= & -\frac{1}{4 \gamma_{1}}\left(\int_{0}^{\infty}\left\langle|\boldsymbol{\zeta}|^{2}\left(|\zeta|^{2}-\zeta_{n}^{2}\right) B \phi^{\mathrm{S}}\right\rangle d \eta-\frac{4}{15}\left\langle|\boldsymbol{\zeta}|^{4}\left(D_{1}-\frac{1}{7}|\zeta|^{2} D_{2}\right) B\right\rangle\right. \\
& \left.+\left.2\left\langle\zeta_{n}\left(|\boldsymbol{\zeta}|^{2}-\zeta_{n}^{2}\right)\left(D_{1}+\frac{1}{2}\left(|\boldsymbol{\zeta}|^{2}-3 \zeta_{n}^{2}\right) D_{2}\right) \phi^{\mathrm{S}}\right\rangle\right|_{\eta=0}-\int_{0}^{\infty}\left\langle\left(|\zeta|^{2}-\zeta_{n}^{2}\right)^{2} \phi^{\mathrm{S}-} \frac{\partial \phi^{\mathrm{S}}}{\partial \zeta_{n}}\right\rangle d \eta\right), \\
a_{2}= & a_{1}+a_{3}, \\
d_{4}= & \frac{2}{5} \frac{\gamma_{1}}{\gamma_{2}} a_{4}, \\
d_{3}= & \frac{4}{5 \gamma_{2}}\left(-\gamma_{3} K_{1}-K_{1} \int_{0}^{\infty}\left\langle|\zeta|^{2} \phi^{\mathrm{T}}\right\rangle d \eta+\frac{1}{2} \int_{0}^{\infty}\left(2 \eta+d_{1}\right)\left\langle\left(|\zeta|^{2}-\zeta_{n}^{2}\right)\left(|\zeta|^{2}-\frac{5}{2}\right) \phi^{\mathrm{C}}\right\rangle d \eta+\frac{2}{15}\left\langle|\boldsymbol{\zeta}|^{4} A F\right\rangle\right. \\
& \left.+\frac{1}{2} \int_{0}^{\infty}\left\langle\left[\left(|\zeta|^{2}-\zeta_{n}^{2}\right) \phi^{\mathrm{C}}-|\boldsymbol{\zeta}|^{2} A\right] \phi^{\mathrm{T}-}\right\rangle d \eta\right),
\end{aligned}
$$


TABLE 2. Dimensionless transport coefficients $\gamma_{1} \sim \gamma_{3}$ and $\gamma_{6}$ (the data taken from [10]).

\begin{tabular}{|c|c|c|c|c|c|c|c|}
\hline & definition & BGK & hard sphere & & definition & BGK & hard sphere \\
\hline$\gamma_{1}$ & $\frac{2}{15}\left\langle|\zeta|^{4} B\right\rangle$ & 1 & 1.270042427 & $\gamma_{3}$ & $\frac{2}{15}\left\langle|\zeta|^{4} A B\right\rangle$ & 1 & 1.947906335 \\
\hline$\gamma_{2}$ & $\frac{4}{15}\left\langle|\zeta|^{4} A\right\rangle$ & 1 & 1.922284066 & $\gamma_{6}$ & $\frac{1}{15}\left\langle|\boldsymbol{\zeta}|^{4}\left(D_{1}+\frac{3}{7}|\boldsymbol{\zeta}|^{2} D_{2}\right) B\right\rangle$ & 1 & 1.419423836 \\
\hline
\end{tabular}

$$
\begin{aligned}
d_{5}= & 2 d_{3}+\frac{8}{5 \gamma_{2}}\left(\int_{0}^{\infty}\left\langle|\zeta|^{2} A \phi^{\mathrm{T}}\right\rangle d \eta-\frac{2}{15}\left\langle|\zeta|^{4} A F\right\rangle-\frac{1}{2} \int_{0}^{\infty}\left\langle\left(|\zeta|^{2}-\zeta_{n}^{2}\right) \phi^{\mathrm{T}-} \frac{\partial \phi^{\mathrm{T}}}{\partial \zeta_{n}}\right\rangle d \eta\right), \\
a_{6}= & a_{4}+k_{0} \frac{\gamma_{3}}{\gamma_{1}}-\frac{1}{\gamma_{1}} \int_{0}^{\infty} \eta\left\langle\left(|\zeta|^{2}-\zeta_{n}^{2}\right)\left(|\zeta|^{2}-\frac{5}{2}\right) \phi^{\mathrm{S}}\right\rangle d \eta-\frac{1}{4 \gamma_{1}} \int_{0}^{\infty}\left\langle|\zeta|^{2}\left(|\zeta|^{2}-\zeta_{n}^{2}\right) B \phi^{\mathrm{C}}\right\rangle d \eta \\
& +\frac{1}{4 \gamma_{1}} \int_{0}^{\infty}\left\langle\left(|\zeta|^{2}-\zeta_{n}^{2}\right)^{2} \phi^{\mathrm{S}-} \frac{\partial \phi^{\mathrm{C}}}{\partial \zeta_{n}}\right\rangle d \eta, \\
a_{5}= & -a_{4}+a_{6}-\frac{\gamma_{3}}{\gamma_{1}} k_{0}+\frac{1}{\gamma_{1}} \int_{0}^{\infty} \eta\left\langle\left(|\zeta|^{2}-\zeta_{n}^{2}\right)\left(|\zeta|^{2}-\frac{5}{2}\right) \phi^{\mathrm{S}}\right\rangle d \eta .
\end{aligned}
$$

Here $\phi^{\mathrm{T}}\left(\eta, \zeta_{n},|\boldsymbol{\zeta}|\right), \zeta_{t} \phi^{\mathrm{S}}\left(\eta, \zeta_{n},|\boldsymbol{\zeta}|\right)$, and $\zeta_{t} \phi^{\mathrm{C}}\left(\eta, \zeta_{n},|\boldsymbol{\zeta}|\right)\left(\zeta_{t}=\zeta_{i} t_{i}\right)$ are the solution of the classical temperature-jump, shear-slip, and thermal creep problems occurring at the first order of the Knudsen number, namely the solution of (12) for $\alpha=\mathrm{T}, \mathrm{S}$, and $\mathrm{C}$ with

$$
\begin{aligned}
& I^{\mathrm{T}}=0, \quad b_{i}^{\mathrm{T}}=0, \quad c^{\mathrm{T}}=d_{1}, \quad g^{\mathrm{T}}=\zeta_{n} A(|\zeta|), \\
& I^{\mathrm{S}}=0, \quad b_{i}^{\mathrm{S}}=-k_{0} t_{i}, \quad c^{\mathrm{S}}=0, \quad g^{\mathrm{S}}=\zeta_{n} \zeta_{t} B(|\boldsymbol{\zeta}|), \\
& I^{\mathrm{C}}=0, \quad b_{i}^{\mathrm{C}}=-K_{1} t_{i}, \quad c^{\mathrm{C}}=0, \quad g^{\mathrm{C}}=\zeta_{t} A(|\boldsymbol{\zeta}|) .
\end{aligned}
$$

It should be mentioned that the coefficients $b_{1}$ and $b_{2}$ are given by definition [10] as

$$
b_{1}=-\frac{1}{4} \int_{0}^{\infty}\left\langle\left(|\zeta|^{2}-\zeta_{n}^{2}\right) \phi^{\mathrm{S}}\right\rangle d \eta, \quad b_{2}=-\frac{1}{2} \int_{0}^{\infty}\left\langle\left(|\zeta|^{2}-\zeta_{n}^{2}\right) \phi^{\mathrm{C}}\right\rangle d \eta .
$$

As explicitly indicated in the above equation, $A, B, D_{1}, D_{2}$ and $F$ are isotropic functions of $\zeta$, which are defined as the solution of the following linear integral equations:

$$
\begin{aligned}
& \mathscr{L}\left[\zeta_{i} A\right]=-\zeta_{i}\left(|\zeta|^{2}-\frac{5}{2}\right) \quad \text { with }\left\langle|\zeta|^{2} A\right\rangle=0, \quad \mathscr{L}\left[\left(\zeta_{i} \zeta_{j}-\frac{1}{3}|\zeta|^{2} \delta_{i j}\right) B\right]=-2\left(\zeta_{i} \zeta_{j}-\frac{1}{3}|\zeta|^{2} \delta_{i j}\right), \\
& \mathscr{L}\left[\left(\zeta_{i} \zeta_{j}-\frac{1}{3}|\zeta|^{2} \delta_{i j}\right) F\right]=\left(\zeta_{i} \zeta_{j}-\frac{1}{3}|\zeta|^{2} \delta_{i j}\right) A, \\
& \mathscr{L}\left[\left(\zeta_{i} \delta_{j k}+\zeta_{j} \delta_{i k}+\zeta_{k} \delta_{i j}\right) D_{1}+\zeta_{i} \zeta_{j} \zeta_{k} D_{2}\right]=\gamma_{1}\left(\zeta_{i} \delta_{j k}+\zeta_{j} \delta_{i k}+\zeta_{k} \delta_{i j}\right)-\zeta_{i} \zeta_{j} \zeta_{k} B \quad \text { with }\left\langle|\zeta|^{2}\left(5 D_{1}+|\zeta|^{2} D_{2}\right)\right\rangle=0,
\end{aligned}
$$

and $\gamma_{1} \sim \gamma_{3}$ and $\gamma_{6}$ are dimensionless transport coefficients, which are constants defined as their integral. The specific values of $\gamma$ 's depend on the molecular model and are listed in Table 2.

We stress that the identities (14)-(23) are general and hold for various intermolecular potential models (the inversepower law, Lennard-Jones, VHS [17], VSS [18], etc.) and boundary conditions. ${ }^{4}$ Moreover, they show that the complete data set of the slip and jump coefficients can be obtained, once we have the solutions of the three classical half-space problems $\phi^{\mathrm{T}}, \phi^{\mathrm{S}}$, and $\phi^{\mathrm{C}}$. Since the accurate numerical method to solve these problems $[12,13]$ has been established in the late 1980 s, we have recomputed the problems using the same method. Then, from the identities (14)(23), we obtain the complete data set of the slip and jump coefficients for a hard-sphere gas with diffuse reflection condition. The results are listed in Table 3. Accuracy of the data of $K_{1}$ and $b_{2}$ has been improved from those given in $[9,10]$ at the last decimal because of the recomputation with a refined grid. Incidentally, we have also confirmed that the data for the BGK model in Table 1 were recovered by the same procedure from the identities (14)-(23).

\footnotetext{
${ }^{4}$ See $[11,16]$ for the class of the boundary conditions to which the symmetric relation can be applied. In addition, it should be noted that the scattering kernel is locally isotropic and that the accommodation coefficient is $O(1)$. These are basic assumptions that are made in Sone's theory of the present concern $[9,10]$. For the boundary with accommodation coefficient of $O(\varepsilon)$, e.g., the (nearly) specular reflection boundary, the slip/jump condition is known to be qualitatively different. See, e.g., Y. Sone and K. Aoki, Phys. Fluids 20, 571 (1977) and K. Aoki, T. Inamuro, and Y. Onishi, J. Phys. Soc. Jpn 47, 663 (1979).
} 
TABLE 3. Slip and jump coefficients for a hard-sphere gas on the diffuse reflection boundary.

\begin{tabular}{|c|c|c|c|c|c|c|c|c|c|}
\hline \multicolumn{2}{|c|}{ first-order slip \& jump } & \multicolumn{4}{|c|}{ second-order slip } & \multicolumn{4}{|c|}{ second-order jump } \\
\hline$k_{0}$ & -1.2540 & $a_{1}$ & 0.9039 & $a_{4}$ & 0.0330 & $d_{3}$ & 0.4992 & $b_{1}$ & 0.1068 \\
\hline$K_{1}$ & -0.6465 & $a_{2}$ & 0.6601 & $a_{5}$ & 0.2336 & $d_{4}$ & 0.0087 & $b_{2}$ & 0.4782 \\
\hline$d_{1}$ & 2.4001 & $a_{3}$ & -0.2438 & $a_{6}$ & -1.9987 & $d_{5}$ & 4.6181 & & \\
\hline
\end{tabular}
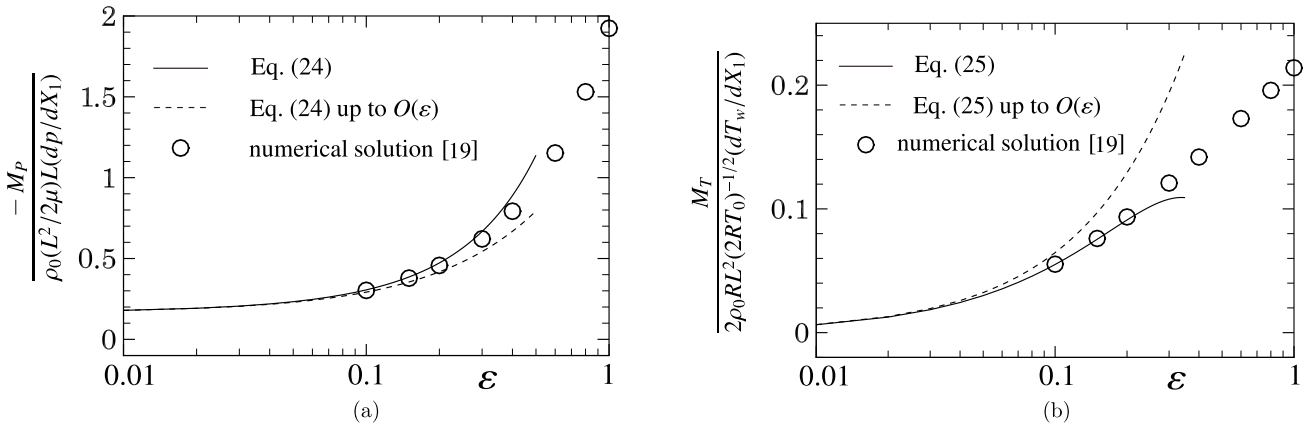

FIGURE 1. Mass flow rate per unit width in a straight channel. (a) Poiseuille flow, (b) thermal transpiration.

\section{SIMPLE EXAMPLES}

We show a few examples (see, e.g., [10]) for which the analytical solution is easily obtained by the slip flow theory.

\section{Mass flow rate of the Poiseuille flow and thermal transpiration}

Consider a slightly rarefied gas in a channel or tube. The flow that is induced by imposing a (small) uniform gradient of pressure along the channel or tube is called the Poiseuille or Hagen-Poiseuille flow, while the flow when a (small) uniform gradient of temperature is imposed along the channel or tube wall is called the thermal transpiration. The Poiseuille and Hagen-Poiseuille flows are commonly observed, while the thermal transpiration is specific to a rarefied gas. The $X_{1}$-direction of the space coordinates $X_{i}$ will be taken along the channel or tube. The quantity with subscript $P$ and $T$ denotes that for Poiseuille (or Hagen-Poiseuille) flow and thermal transpiration, respectively. The pressure and the wall temperature will be denoted by $p$ and $T_{w}$, respectively.

First consider the case of a straight channel. Let $L$ be the separation distance between the channel walls. Mass flow $(M, 0,0)$ per unit time for unit spanwise width can be computed to yield $[10,19]$

$$
\begin{aligned}
& \frac{M_{P}}{\rho_{0}\left(L^{2} / 2 \mu\right) L\left(d p / d X_{1}\right)}=-\frac{1}{6}+k_{0} \varepsilon-2\left(a_{1}-2 b_{1}\right) \varepsilon^{2}, \\
& \frac{M_{T}}{2 \rho_{0} R L^{2}\left(2 R T_{0}\right)^{-1 / 2}\left(d T_{w} / d X_{1}\right)}=-\left(K_{1}+2 b_{2} \varepsilon\right) \varepsilon .
\end{aligned}
$$

It should be noted that the expansion has been completed in the above expression (the third and higher order terms do not appear). However, the above should be recognized as the asymptotic solution and can be applied only for small $\varepsilon$. The results for a hard-sphere gas are plotted and compared with the direct numerical solution [19] in Fig. 1.

Next, consider the case of a straight tube with a circular cross-section. Let $L$ be the radius of the tube. Mass flow $(M, 0,0)$ per unit time can be computed [20] to yield

$$
\begin{aligned}
& \frac{M_{P}}{\rho_{0}\left(\pi L^{3} / 2 \mu\right) L\left(d p / d X_{1}\right)}=-\frac{1}{4}+k_{0} \varepsilon+\left(4 b_{1}-a_{1}-\frac{a_{2}}{2}\right) \varepsilon^{2}+\cdots, \\
& \frac{M_{T}}{2 \rho_{0} R\left(\pi L^{3}\right)\left(2 R T_{0}\right)^{-1 / 2}\left(d T_{w} / d X_{1}\right)}=-K_{1} \varepsilon-\left(2 b_{2}-\frac{a_{5}}{2}\right) \varepsilon^{2}+\cdots .
\end{aligned}
$$

The results for a hard-sphere gas are plotted and compared with the numerical solution [21] in Fig. 2. 


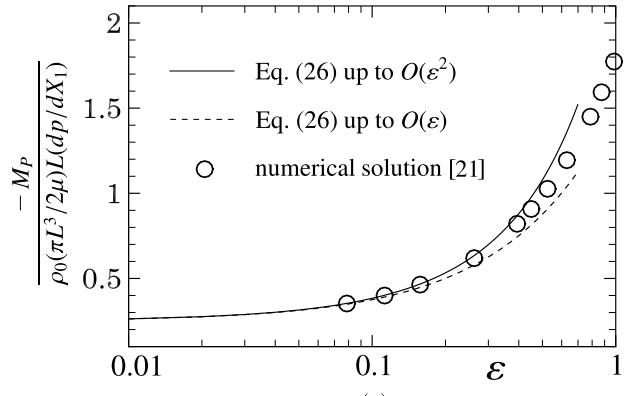

(a)

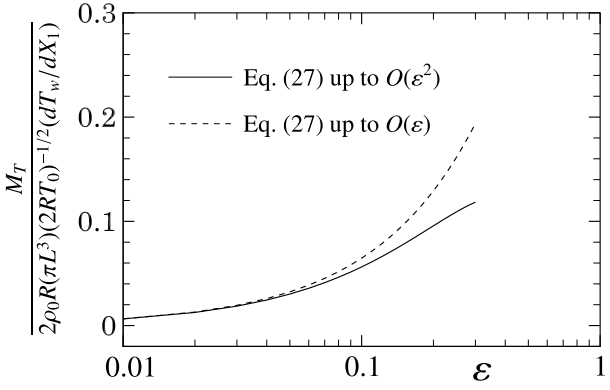

(b)

FIGURE 2. Mass flow rate in a straight tube with a circular cross-section. (a) Hagen-Poiseuille flow, (b) thermal transpiration.

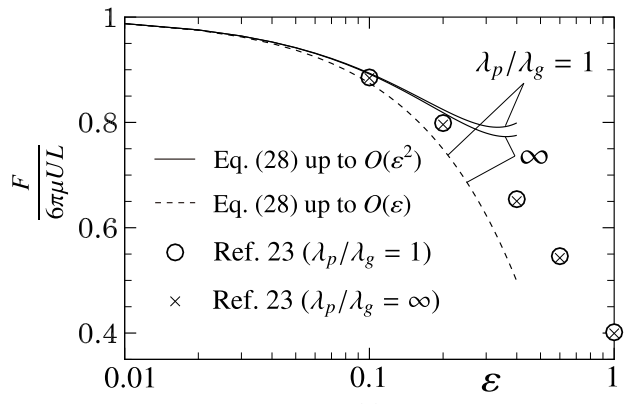

(a)

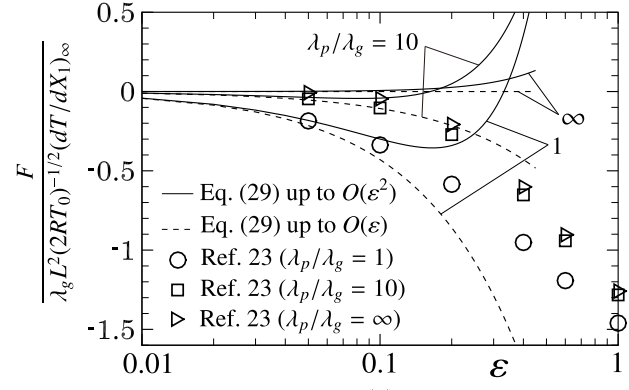

(b)

FIGURE 3. Forces acting on a spherical particle. (a) Drag force, (b) thermal force.

\section{Drag and thermal forces acting on a spherical particle}

Consider a spherical particle of radius $L$ suspended in a slightly rarefied gas. The thermal conductivities of the particle and the gas will be denoted by $\lambda_{p}$ and $\lambda_{g}$, respectively. The origin of the space coordinates $X_{i}$ is the center of the sphere.

The drag force $(F, 0,0)$ acting on the sphere in a slow uniform gas flow $(U, 0,0)$ can be computed $[10,22]$ to yield

$$
\frac{F}{6 \pi \mu U L}=1+k_{0} \varepsilon+\left(3 k_{0}^{2}-3 a_{1}+2 a_{3}+2 b_{1}+\frac{8}{5} \frac{\operatorname{Pr} K_{1}^{2}}{\lambda_{p} / \lambda_{g}+2}\right) \varepsilon^{2}+\cdots,
$$

where $\mu$ is the viscosity, $\operatorname{Pr}\left(=\gamma_{1} / \gamma_{2}\right)$ is the Prandtl number. This formula is taken from [10], but is simplified by using (14) and (18). The results for a hard-sphere gas are plotted and compared with the direct numerical solution [23] in Fig. 3(a).

When there is a small temperature gradient in the background gas, namely when the temperature of the gas in the absence of the sphere is given by $T_{0}+\left(d T / d X_{1}\right)_{\infty} X_{1}$, the sphere is subjected to a force from the gas (the thermal force). The thermal force $(F, 0,0)$ is computed $[10,22]$ to yield

$$
\begin{gathered}
\frac{F}{\lambda_{g} L^{2}\left(2 R T_{0}\right)^{-1 / 2}\left(d T / d X_{1}\right)_{\infty}}=\frac{48 \pi}{5} \operatorname{Pr}\left(\frac{K_{1}}{2+\lambda_{p} / \lambda_{g}} \varepsilon+\left(a_{4}-\frac{C}{2+\lambda_{p} / \lambda_{g}}\right) \varepsilon^{2}\right)+\cdots \\
C=-b_{2}-3 K_{1} k_{0}+3 a_{4}-a_{5}-a_{6}+\frac{2 K_{1}}{2+\lambda_{p} / \lambda_{g}}\left(d_{1} \frac{\lambda_{p}}{\lambda_{g}}-\frac{4}{5 \gamma_{2}} \int_{0}^{\infty} H_{B} d \eta\right), \\
\int_{0}^{\infty} H_{B} d \eta=1.2765 \text { (hard sphere), } 0.41556(\mathrm{BGK}) .
\end{gathered}
$$

The results for a hard-sphere gas are plotted and compared with the direct numerical solution [23] in Fig. 3(b). When $\lambda_{p} / \lambda_{g}$ is finite, the first term is dominant, and the thermal force is acting in the direction opposite to the imposed 
gradient of temperature (the usual thermophoresis) because $K_{1}<0$. When $\lambda_{p} / \lambda_{g}$ is very large or ideally infinite, only the $a_{4} \varepsilon^{2}$ remains in the parenthesis, and the thermal force is acting in the same direction as the temperature gradient (the negative thermophoresis $[24,14]$ ) because $a_{4}>0$. Therefore, the direction of the thermophoresis may change depending on the material of the particle, though the negative thermophoresis has not been verified experimentally.

The reversal of the thermal force is due to the change of its physical mechanism. In the usual thermophoresis, the surface temperature of the sphere is nonuniform because of the finite thermal conductivity of the particle. The nonuniform surface temperature, then, induces the thermal creep flow and is subjected to the reaction from the gas. The first term of (29a) represents this effect. On the other hand, in the negative thermophoresis, the surface temperature is uniform because of the infinite thermal conductivity of the particle, thus the thermal creep effect is absent. Then, the higher-order effect of the thermal-stress slip is dominant, inducing a flow (thermal-stress slip flow) in the opposite to the thermal creep. Thus, the reaction from the gas is in the opposite direction to the case of the thermal creep effect.

\section{ACKNOWLEDGMENTS}

The present work is supported in part by JSPS KAKENHI (No. 23360083).

\section{REFERENCES}

1. P. L. Bhatnagar, E. P. Gross, and M. Krook, Phys. Rev. 94, 511-525 (1954).

2. P. Welander, Ark. Fys. 7, 507-553 (1954).

3. Y. Sone, "Asymptotic theory of flow of rarefied gas over a smooth boundary I," in Rarefied Gas Dynamics, edited by L. Trilling and H. Y. Wachman, Academic, New York, 1969, Vol. I, pp. 243-253.

4. Y. Sone and K. Aoki, Transp. Theory Stat. Phys. 16, 189-199 (1987).

5. C. Aubert and S. Colin, Microscale Thermophysical Engineering 5, 41-54 (2001).

6. J. Maurer, P. Tabeling, P. Joseph, and H. Willaime, Phys. Fluids 15, 2613-2621 (2003).

7. S. Colin, P. Lalonde, and R. Caen, Heat Transfer Engineering 25, 23-30 (2004).

8. N. Xiao, J. Elsnab, and T. Ameel, International Journal of Thermal Sciences 48, 243-251 (2009).

9. Y. Sone, Kinetic Theory and Fluid Dynamics, Birkhäuser, Boston, 2002; Supplementary notes and errata are available from http://hdl.handle.net/2433/66099.

10. Y. Sone, Molecular Gas Dynamics, Birkhäuser, Boston, 2007; Supplementary notes and errata are available from http://hdl.handle.net/2433/66098.

11. S. Takata, J. Stat. Phys. 136, 751-784 (2009).

12. Y. Sone, T. Ohwada, and K. Aoki, Phys. Fluids A 1, 363-370 (1989).

13. T. Ohwada, Y. Sone, and K. Aoki, Phys. Fluids A 1, 1588-1599 (1989).

14. T. Ohwada and Y. Sone, Eur. J. Mech. B-Fluids 11, 389-414 (1992).

15. S. Takata, Phys. Fluids 21, 112001 (2009).

16. S. Takata and M. Hattori, J. Stat. Phys. 147, 1182-1215 (2012).

17. G. A. Bird, "Monte-Carlo simulation in an engineering context," in Rarefied Gas Dynamics, edited by S. S. Fisher, AIAA, New York, 1981, Vol. I, pp. 239-255.

18. K. Koura and H. Matsumoto, Phys. Fluids A 3, 2459-2465 (1991).

19. T. Ohwada, Y. Sone, and K. Aoki, Phys. Fluids A 1, 2042-2049 (1989).

20. H. Funagane and S. Takata, Fluid Dynamics Research (in press).

21. S. K. Loyalka and S. A. Hamoodi, Phys. Fluids A 2, 2061-2065 (1990), erratum ibid A 3, 2825 (1991).

22. Y. Sone and K. Aoki, "Forces on a spherical particle in a slightly rarefied gas," in Rarefied Gas Dynamics: Progress in Astronautic and Aeronautics, Vol. 51, edited by J. L. Potter, AIP, New York, 1977, pp. 417-433.

23. S. Takata and Y. Sone, Eur. J. Mech., B/Fluids 14, 487-518 (1995).

24. Y. Sone and K. Aoki, "Negative thermophoresis: Thermal stress slip flow aournd a spherical particle in a rarefied gas," in Rarefied Gas Dynamics: Progress in Astronautics and Aeronautics, Vol. 74, edited by S. S. Fisher, AIP, New York, 1981, pp. 489-503. 
Copyright of AIP Conference Proceedings is the property of American Institute of Physics and its content may not be copied or emailed to multiple sites or posted to a listserv without the copyright holder's express written permission. However, users may print, download, or email articles for individual use. 